Marlies Ostermann Michael Joannidis

\section{Biomarkers for AKI improve clinical practice:}

no
Received: 28 October 2014

Accepted: 28 October 2014

Published online: 12 November 2014

(C) Springer-Verlag Berlin Heidelberg and ESICM 2014

For a contrasting viewpoint, please go to

doi:10.1007/s00134-014-3530-2.

\section{Ostermann}

Department of Critical Care and Nephrology, King's College

London, Guy's and St Thomas' Foundation Hospital, Westminster Bridge Road, London SE1 7EH, UK

\section{Joannidis (®)}

Division of Intensive Care and Emergency Medicine,

Department of Internal Medicine I, Medical University Innsbruck, Anichstrasse 35, 6020 Innsbruck, Austria

e-mail: michael.joannidis@i-med.ac.at

Tel.: +4351250424181

Despite recent developments in definition and staging, the diagnosis of (AKI) acute kidney injury is still based on oliguria and/or an increase in serum creatinine [1]. Consequently, research in the last decade has focussed on the discovery and validation of more specific and sensitive markers of tubular damage/functional impairment. The most advanced biomarkers promise to identify patients at risk of AKI, diagnose AKI earlier than conventional tests and prognosticate risk of progression, including need for (RRT) renal replacement therapy [2-16]. The hope is that, with such an approach and more timely and relevant interventions, the outcome of patients with AKI can be improved. Commercial biomarker kits are now available in most countries. However, like any other new diagnostic test, these novel AKI biomarkers need to add value above and beyond currently available tools, before being incorporated into routine clinical practice. It is therefore essential to have a close look at the expectations of AKI biomarkers and their actual performance and potential role in practice.

1. Can novel AKI biomarkers help to identify high-risk patients? In principle, it is appealing to identify highrisk patients, especially if this provides an opportunity to intervene and prevent the event of interest. In the case of AKI, the currently recommended preventative strategies include optimisation of haemodynamics and volume status and avoidance of further harm, i.e. avoidance of nephrotoxic drugs, contrast media, or starches [1]. One could easily argue that this should be done in any critically ill patient, regardless of whether or not they are at high risk of AKI, and that therefore the information from a new biomarker test is redundant.

Ideally, a test to identify high-risk patients should be as reliable as possible to avoid false positive and false negative results. Experience from other clinical areas has taught that non-selected use of biomarkers like prostatespecific antigen or d-dimer in an unselected population produces a high number of false positive results and can potentially lead to harmful and unnecessary interventions. In the case of AKI, the area under the receiver operating characteristics curves of some biomarkers look impressive but the clinical application of the results to individual patients is hampered by wide overlap between groups. Similarly, biomarker studies vary in their cut-offs between positive and negative results (Table 1). As a result, potential harm may occur if important interventions (i.e. computed tomography with contrast or necessary administration of aminoglycosides) are being delayed or withheld based on false positive biomarker results.

Some biomarkers, for instance plasma neutrophil gelatinase-associated lipocalin (NGAL), reflect a general degree of severity of disease, rather than being specific for kidney injury $[4,17,18]$. In this case, the role of kidneyspecific preventative measures is questionable. 


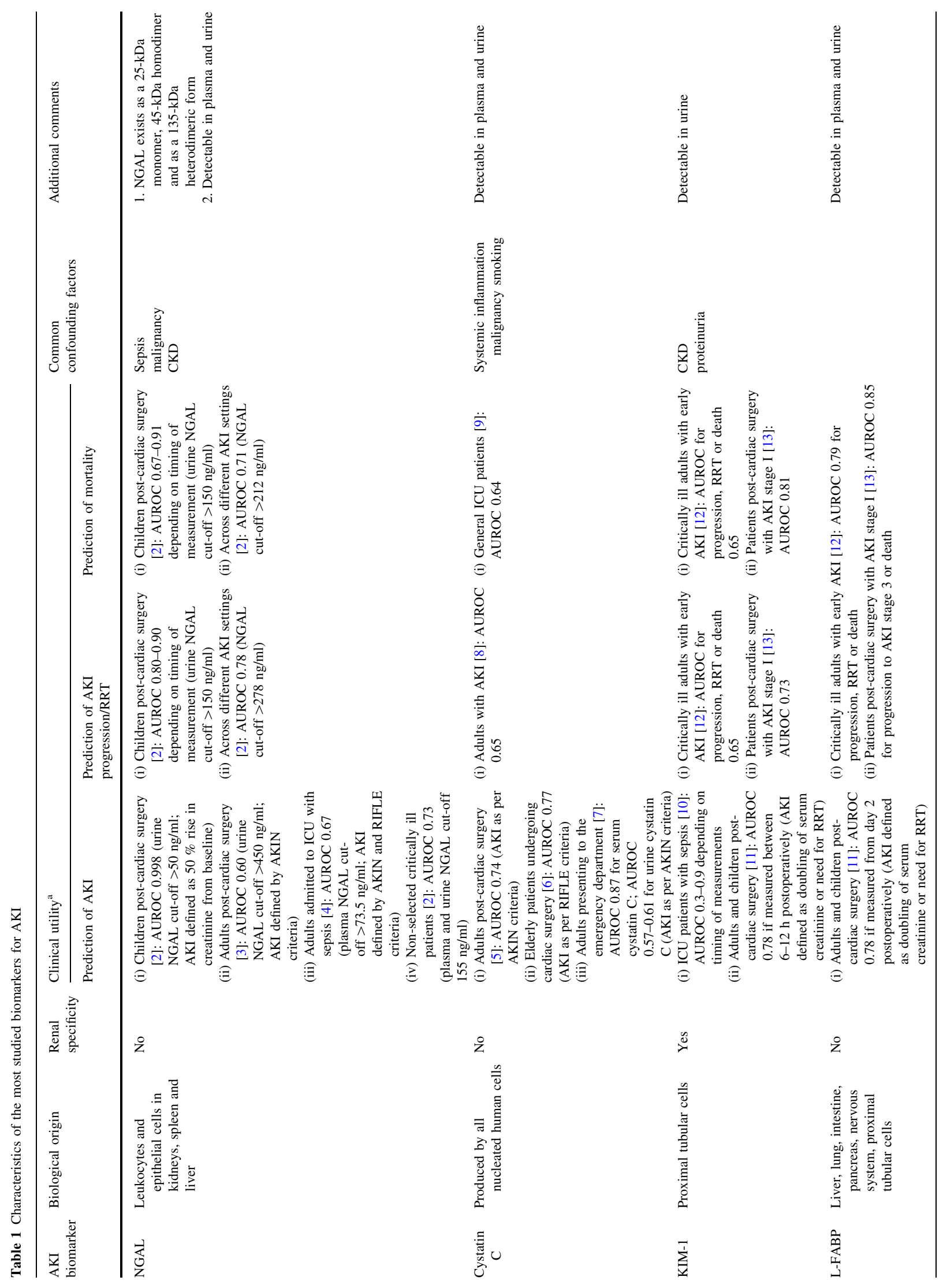


Finally, the performance of most biomarkers for AKI is better in selected patient groups at high risk of AKI. For instance, the recently FDA approved Nephrocheck ${ }^{\circledR}$, which measures two cell cycle arrest markers, insulinlike growth factor binding protein 7 and tissue metalloproteinase-2, was evaluated and validated in critically ill patients in the intensive care unit (ICU) $[14,16]$. Whether it performs equally well in unselected cohorts like patients in non-ICU wards or the emergency department remains to be seen.

2. Can novel AKI biomarkers diagnose AKI earlier than traditional tests? Several biomarkers have been shown to indicate the onset of AKI before serum creatinine rises. The results are most impressive in paediatric cohorts without comorbidities suffering from an illness with a defined onset of AKI, for instance in children after cardiac surgery [2] (Table 1). In more heterogeneous populations, where the onset of renal injury is not known (i.e. patients with septic shock), the performance of some biomarkers to detect AKI earlier was equivalent to clinical evaluation and standard laboratory measurements but not significantly better [3, 4].

It is generally assumed that an earlier diagnosis of AKI automatically translates into earlier treatment and better patient outcome. Unfortunately, there is currently no evidence that this is indeed the case. Also, there is no specific therapy for AKI. To date, the management of AKI is supportive with emphasis on optimisation of haemodynamic and fluid status and avoidance of further nephrotoxic insults [1], i.e. strategies which should form part of good critical care in all patients at any time.

3. Can novel AKI biomarkers identify patients who need RRT? Predicting whether patients will progress to needing RRT confronts us with a similar conflict as predicting who will develop AKI. To date, there are no interventions beyond treatment of the underlying illness, attention to detail and good medical care which prevent progression of AKI or induce recovery. Although it may appear attractive to know which patient will need RRT, this knowledge is unlikely to change clinical practice, especially since there is no evidence that starting RRT earlier before it is needed by current criteria is beneficial [19]. However, knowing whether a patient on RRT has recovered sufficient native renal function so that RRT can be stopped would be useful but, to date, none of the available biomarkers are capable of doing so.

4. Do novel AKI biomarkers improve patient outcome? No biomarker has yet shown the efficacy of any intervention based on increased biomarkers. The only intervention study using biomarkers to guide treatment was negative [20]. Therefore, the claim that biomarker use benefits patients and improves outcome remains unproven. 
5. When should AKI biomarkers be measured? One of the difficulties in utilizing AKI biomarkers has been to identify which patients would benefit most. Some studies advertise the use of biomarkers in situations where the outcome already seems predictable based on standard parameters, such as clinical appraisal and oliguria. Clearly, in this situation, there is little added benefit. Similarly, indiscriminate application of biomarkers in patients at low risk of AKI would also render the biomarker useless, as well as unnecessarily increase healthcare costs.

There are additional methodological problems in biomarker research leaving clinicians with uncertainty. First, in the majority of studies, the performance of novel biomarkers was judged by comparison with serum creatinine and oliguria, two markers which are affected by confounding factors and often proclaimed to be not sufficiently kidney-specific. Second, studies vary in the chosen cut-offs to establish thresholds for negative and positive predictive events related to AKI (Table 1). Third, there is uncertainty regarding the exact laboratory method, the assay platform and sampling conditions and whether biomarker levels should be normalised for urinary creatinine $[17,18]$. Fourth, like creatinine, several novel biomarkers of AKI are themselves not renal-specific and confounded by common comorbid conditions, for instance sepsis and chronic kidney disease (Table 1). Finally, most biomarkers demonstrate a dynamic pattern reflective of the molecular and cellular events that occur throughout the clinical phases of AKI. It is therefore likely that a panel of different biomarkers and multiple measurements will be necessary rather than a single test. Whether the associated costs and turn-around time are realistic in clinical practice is questionable.

We acknowledge that some studies have shown very impressive results and agree that biomarkers have the potential to transform the way we diagnose and treat patients with AKI. However, at this stage, there are still too many unresolved concerns and uncertainties which hamper their clinical applicability.

Conflicts of interest M.O. received speaker's honoraria from Alere, M.J. received speaker's honoraria from Astute Medical.

\section{References}

1. Kidney Disease: Improving Global Outcomes (KDIGO) Acute Kidney Injury Work Group (2012) KDIGO clinical practice guideline for acute kidney injury. Kidney Int 2:1-138

2. Haase M, Bellomo R, Devarajan P, Schlattmann P, Haase-Fielitz A, NGAL Meta-analysis Investigator Group (2009) Accuracy of neutrophil gelatinase-associated lipocalin (NGAL) in diagnosis and prognosis in acute kidney injury: a systematic review and meta-analysis. Am J Kidney Dis 54:1012-1024

3. Wagener G, Gubitosa G, Wang S, Borregaard N, Kim M, Lee HT (2008) Urinary neutrophil gelatinaseassociated lipocalin and acute kidney injury after cardiac surgery. Am J Kidney Dis 52(3):425-433

4. Martensson J, Bell M, Oldner A, Xu S, Venge P, Martling CR (2010) Neutrophil gelatinase-associated lipocalin in adult septic patients with and without acute kidney injury. Intensive Care Med 36:1333-1340

5. Haase M, Bellomo R, Devarajan P, Ma Q, Bennett MR, Möckel M, Matalanis G, Dragun D, Haase-Fielitz A (2009) Novel biomarkers early predict the severity of acute kidney injury after cardiac surgery in adults. Ann Thorac Surg 88:124-130
6. Ristikankare A, Poyhia R, Kuitunen A, Skrifvars $M$, Hämmäinen $P$, Salmenperä M, Suojaranta-Ylinen R (2010) Serum cystatin C in elderly cardiac surgery patients. Ann Thorac Surg 89:689-694

7. Soto K, Coelho S, Rodriques B, Martins H, Frade F, Lopes S, Cunha L, Papoila AL, Devarajan P (2010) Cystatin C as a marker of acute kidney injury in the emergency department. Clin J Am Soc Nephrol 5(10):1745-1754

8. Perianayagam MC, Seabra VF, Tighiouart H, Liangos O, Jaber BL (2009) Serum cystatin C for prediction of dialysis requirement or death in acute kidney injury: a comparative study. Am J Kidney Dis 54:1025-1033

9. Nejat M, Pickering JW, Walker RJ, Westhuyzen J, Shaw GM, Frampton CM, Endre ZH (2010) Urinary cystatin $\mathrm{C}$ is diagnostic of acute kidney injury and sepsis, and predicts mortality in the intensive care unit. Crit Care 14(3):R85

10. Tu Y, Wang H, Suni R, Ni Y, Ma L, Xv F, Hu X, Jiang L, Wu A (2014) Urinary netrin-1 and KIM-1 as early biomarkers for septic acute kidney injury. Ren Fail $26: 1-5$
11. Parikh CR, Thiessen-Philbrook H, Garg AX, Kadiyala D, Shlipak MG, Koyner JL, Edelstein CL, Devarajan P, Patel UD, Zappitelli M, Krawczeski CD, Passik CS, Coca SG, TRIBE-AKI Consortium (2013) Performance of kidney injury molecule-1 and liver fatty acid-binding protein and combined biomarkers of AKI after cardiac surgery. Clin J Am Soc Nephrol 8(7):1079-1088

12. Parr SK, Clark AJ, Bian A, Shintani AK, Wickersham NE, Ware LB, Ikizler TA, Siew ED (2014) Urinary L-FABP predicts poor outcomes in critically ill patients with early acute kidney injury. Kidney Int. doi:10.1038/ki.2014.301

13. Arthur JM, Hill EG, Alge JL, Lewis EC, Neely BA, Janech MG, Tumlin JA, Chawla LS, Shaw AD, SAKInet Investigators (2014) Evaluation of 32 urine biomarkers to predict the progression of acute kidney injury after cardiac surgery. Kidney Int 85(2):431-438

14. Kashani K, Al-Khafaji A, Ardiles T et al (2013) Discovery and validation of cell cycle arrest biomarkers in human acute kidney injury. Crit Care 17:R25 
15. Hoste EA, McCullough PA, Kashani K, 17. Glassford NJ, Schneider AG, Xu S et al Chawla LS, Joannidis M, Shaw AD, Feldkamp T, Uettwiller-Geiger DL, McCarthy P, Shi J, Walker MG, Kellum JA; for the Sapphire Investigators (2014) Derivation and validation of cutoffs for clinical use of cell cycle arrest biomarkers. Nephrol Dial Transplant. pii:gfu292 (Epub ahead of print. PubMed PMID: 25237065)

16. Bihorac A, Chawla LS, Shaw AD et al (2014) Validation of cell-cycle arrest biomarkers for acute kidney injury using clinical adjudication. Am J Respir Crit Care Med 189:932-939
(2013) NGAL and AKI: the end of a myth? Intensive Care Med 39(10):1861-1863
19. Joannidis M, Forni LG (2011) Clinical review: timing of renal replacement therapy. Crit Care 15(3):223 (2013) The nature and discriminatory associated lipocalin in critically ill patients at risk of acute kidney injury. Intensive Care Med 39:1714-1724

20. Endre Z, Walker R, Pickering $\mathrm{J}$ et al (2010) Early intervention with erythropoietin does not affect the outcome of acute kidney injury (the EARLYARF trial). Kidney Int 77:1020-1030 